\title{
Accelerating EUV learning with synchrotron light: Mask roughness challenges ahead
}

\author{
Patrick P. Naulleau, ${ }^{1}$ Kenneth A. Goldberg, ${ }^{1}$ Eric Gullikson, ${ }^{1}$ Iacopo Mochi, ${ }^{1}$ Brittany McClinton, ${ }^{2}$ \\ and Abbas Rastegar ${ }^{3}$ \\ ${ }^{1}$ Center for X-Ray Optics, Lawrence Berkeley National Laboratory, Berkeley, CA 94720 \\ ${ }^{2}$ University of California, Berkeley, CA 94720 \\ ${ }^{3}$ SEMATECH, 257 Fuller Road, Suite 2200, Albany, NY 12203
}

\begin{abstract}
Despite significant progress in the commercialization of extreme ultraviolet (EUV) lithography, many important challenges remain, including in the area of masks. The issue of EUV phase roughness that can arise from either multilayer or capping layer roughness has recently garnered increasing concern. The problem with mask phase roughness is that it couples line-edge roughness (LER) through the formation of image plane speckle. The coupling from phase roughness to LER depends on many factors including roughness magnitude, roughness correlation length, illumination partial coherence, aberrations, defocus, and numerical aperture. Analysis shows that only on the order of 50 pm multilayer roughness may be tolerable at the 22-nm half-pitch node. Results also show that Atomic Force Microscopy (AFM) may not be a suitable method for measuring mask phase roughness due to its sensitivity to the surface only. Capping layer roughness is another significant concern especially given that it has been shown to increase with cleaning cycles. In this case, however, AFM does provide a reasonable metric.
\end{abstract}

Keywords: extreme ultraviolet, lithography, multilayer, surface roughness, line-edge roughness

\section{INTRODUCTION}

Despite significant progress in the commercialization of extreme ultraviolet (EUV) lithography [1], many challenges remain including important challenges in the area of masks. Of these mask challenges, certainly defectivity is the most pressing and well known [2]. Significant progress has been made in this area, but another two orders of magnitude defect reduction is still required to meet current pilot lines goals [2]. Another significant concern for EUV masks is critical dimension uniformity (CDU) and in particular effective CDU at the wafer. The non-telecentric nature of EUV masks due to the requisite off-axis illumination, raises a variety of EUV specific problems. A thorough survey of the various sources of mask-induced CDU has been presented by Gallagher et al. [3].

A third area of significant concern for EUV masks is mask-induced line-edge roughness (LER). Here we summarize mask contributors to LER including mask multilayer roughness, mask capping layer roughness, and mask LER. We also discuss implications on mask requirements, metrology methods, and mask cleaning processes.

\section{MASK MULTILAYER ROUGHNESS}

Roughness that is reproduced throughout a significant portion of the multilayer stack is referred to as replicated surface roughness (RSR). This roughness directly maps to phase roughness in the reflected field which in turn maps to image plane speckle and LER [4-7]. The concern over, this problem has recently increased since its experimental demonstration in printed wafers at EUV [8] in both an microfield exposure tool [9] and a full field EUV alpha tool [3]. The coupling from roughness to LER depends on many factors including roughness magnitude, roughness correlation length, illumination partial coherence, aberrations, defocus, and numerical aperture (NA) [10]. To study this effect, scalar twodimensional aerial image modeling can be used $[6,11]$. In this model, the multilayer roughness is represented as in-plane phase variations at the mask. Accounting for reflection from the Bragg structure and assuming the roughness to be replicated throughout at least the top 10 bilayers of the multilayer, the phase variations are set to two times the multilayer height variations to be modeled. A detailed description of this modeling technique can be found in Ref. [6]. 
As stipulated above, mask phase roughness couples to LER through the generation of image-plane speckle, or random intensity variations. The reason that a pure phase distribution translates to intensity variations at the wafer is that the imaging system itself is band-limited meaning that even an aberration-free system cannot faithfully reproduce the exact electric field and failure to do so causes the magnitude squared of the electric field to no longer be perfectly uniform. The problem becomes even more severe as we deviate from an ideal system by, for example, moving out of focus. Moving out of focus is identical to observing the electric field after propagation a certain distance away from the mask. This process causes points with random phase to mix and interfere causing speckle [12]. Another way to view this process is through a concept known as transport of intensity [13-15] where random wavefront curvatures in one plane causes ray bundle concentration (intensity) variations in another plane.

Figure 1 shows the modeled image plane speckle arising from a mask with $230 \mathrm{pm}$ RSR when imaged using an optical system with a NA of 0.25 . What is being modeled is a clear area on the mask and in the ideal case we would see perfectly uniform intensity at the wafer. Figure 1(a) shows the image intensity at best focus and with a partial coherence of 0.5 yielding an RMS contrast of $0.9 \%$. Figure 1(b) is with $50 \mathrm{~nm}$ defocus and again with a partial coherence of 0.5 yielding an RMS contrast of $6 \%$. Finally, Fig 1 (c) is with $50 \mathrm{~nm}$ defocus and again with a partial coherence of 0.3 yielding an RMS contrast of $9 \%$.

a)

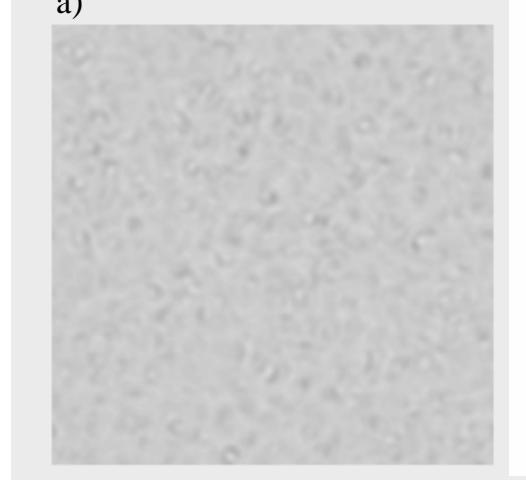

r ıy. 1. ivivucıcu mirage prane specne arising from a mask with $230 \mathrm{pm}$ RSR when imaged using an optical system with a numerical aperture of 0.25 . (a) is at best focus with a partial coherence of 0.5 yielding an RMS contrast of $0.9 \%$. (b) is with $50 \mathrm{~nm}$ defocus with a partial coherence of 0.5 yielding an RMS contrast of $6 \%$. (c) is with $50 \mathrm{~nm}$ defocus with a partial coherence of 0.3 yielding an RMS contrast of $9 \%$.

The effect of roughness and its dependence on focus can be directly visualized using an EUV mask imaging microscope. Figure 2 shows images from the SEMATECH Berkeley Actinic Inspection Tool (AIT) [16] imaging a typical EUV mask using an equivalent NA of 0.25 and an effective partial coherence of approximately 0.15 . The image labels represent the defocus in $\mathrm{nm}$. As the defocus is increased, so is the speckle magnitude. Additionally it is evident that the line contrast decreases at the same time causing the impact on LER to be even greater. The impact of the mask roughness on the wafer plane LER has also been directly experimentally verified using the SEMATECH Berkeley MET. To accentuate the effect, a small partial coherence $(0.05)$ and large defocus $(100 \mathrm{~nm})$ was used [8]. Figure 3 shows printed line edge data for a single line edge exposed three separate times. The total analyzed line length is approximately $1 \mu \mathrm{m}$. The correlation of the line edge data from exposure to exposure is a measure of the mask contributions since any resist contributions would certainly be expected to be uncorrelated from exposure to exposure. These effects have further been verified in a full field Alpha Demo Tool using a conventional discharge source and a partial coherence of 0.5 [3].

The experimental results on the effect of mask roughness obtained on the SEMATECH Berkeley MET have been used to verify a model we have developed to predict this effect [8]. This model has subsequently been used to predict sensitivity of the mask roughness effect to a variety of mask and optical system parameters and to estimate future mask requirements [10]. The most obvious mask parameter to consider is roughness. Figure 4 shows the computed image plane line-width roughness (LWR) as a function of RSR for various amounts of defocus. Also assumed is a NA of 0.32, 22-nm half pitch line/space features, a partial coherence of 0.5 , flare of $5 \%$, a roughness correlation length of $100 \mathrm{~nm}$, and a roughness exponent of 1 . The results show that even with an RSR as small as $50 \mathrm{pm}$, we have 1-nm of LWR at 75nm defocus. 

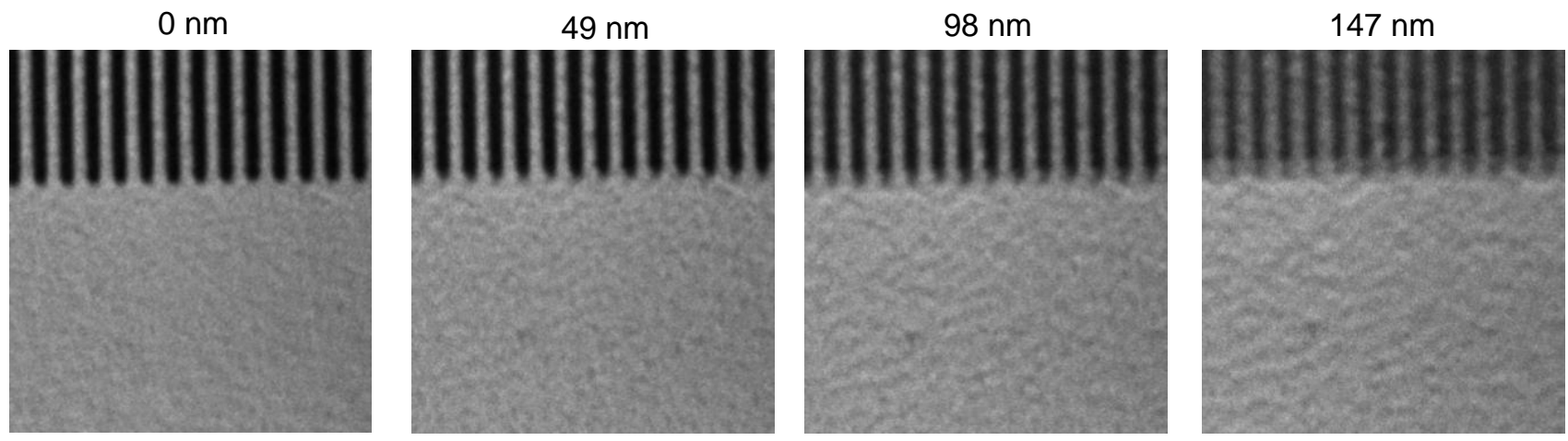

Fig. 2. Demonstration of mask induced speckle using an EUV aerial imaging microscope. The image labels represent the defocus in $\mathrm{nm}$. The predicted speckle contrast increase with defocus is observed.

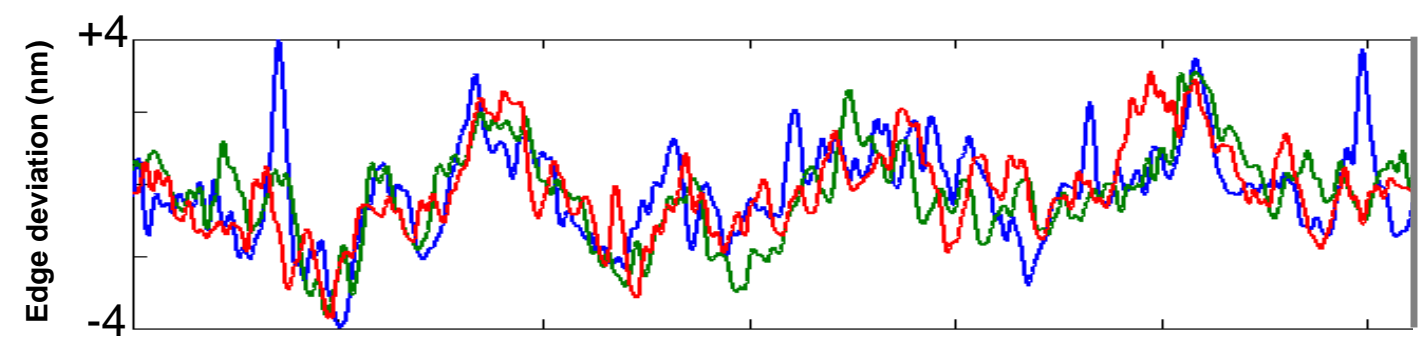

\section{Position along length of line}

Fig. 3. Printed line edge data for a single line edge exposed three separate times. The total analyzed line length is approximately $1 \mu \mathrm{m}$.

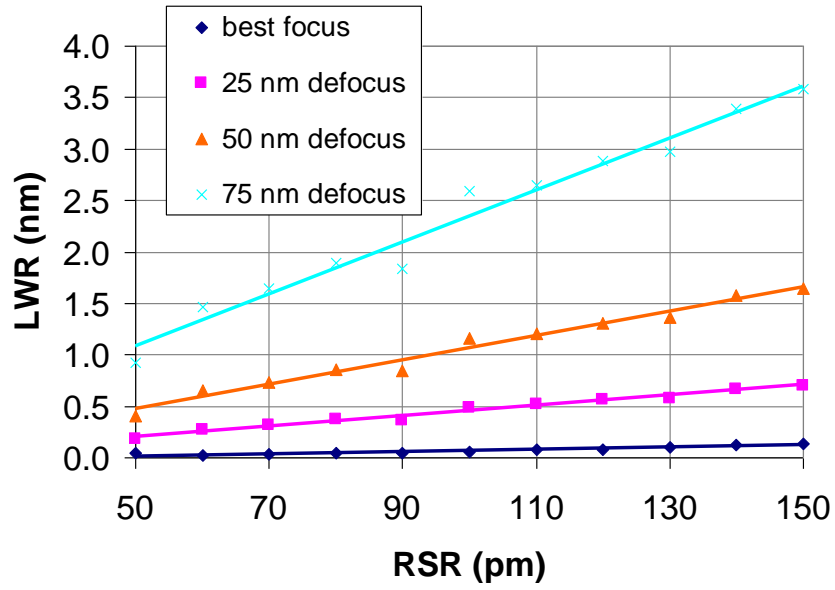

Fig. 4. Computed image plane line-width roughness (LWR) as a function of RSR for various amounts of defocus. Also assumed is a numerical aperture of $0.32,22$-nm half pitch line/space features, a partial coherence of 0.5 , flare of $5 \%$, a roughness correlation length of $100 \mathrm{~nm}$, and a roughness exponent of 1 . 
Certainly the increase in LWR as a function of RSR should be expected. Less obvious, however, is the dependence of the LWR on the correlation length of the RSR. In general one would expect the correlation length to be determined by the coating properties and for magnetron coating the correlation length is typically on the order of $100 \mathrm{~nm}$. Figure 5 shows the effect of the correlation length. Again we assume a NA of 0.32 , a feature size of $22 \mathrm{~nm}$, a partial coherence of 0.5 , flare of $5 \%$, and a roughness exponent of 1 . We also assume an RSR of $100 \mathrm{pm}$. In this case we see peaked behavior where the peak position is determined by the NA of the optic. Unfortunately, the peak occurs very close to the correlation length typically observed in EUV masks.

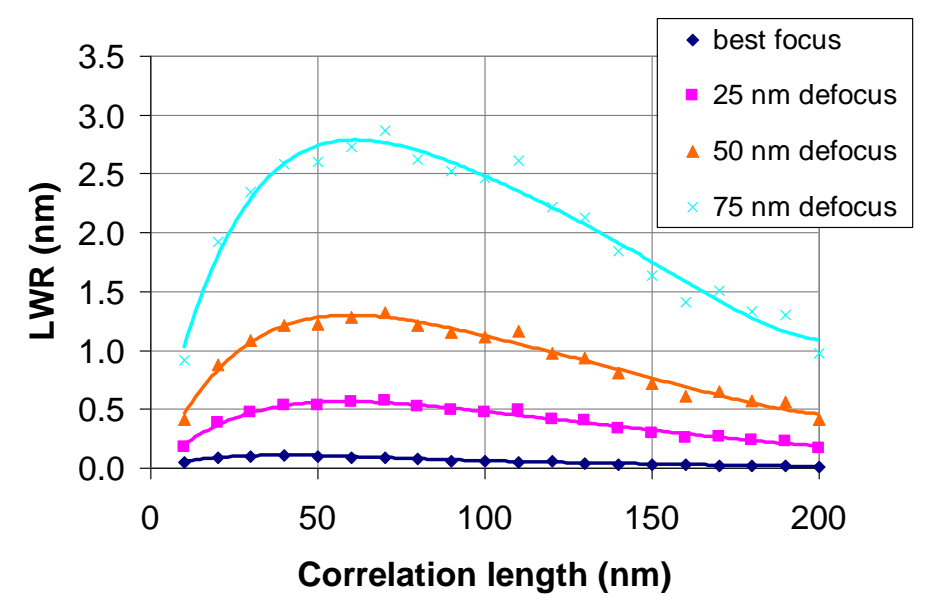

Fig. 5. Effect of RSR correlation length on computed image plane LWR. The LWR behavior is peaked with the peak position being determined by the numerical aperture of the optic. Again we assume a numerical aperture of 0.32 , a feature size of $22 \mathrm{~nm}$, a partial coherence of 0.5 , flare of $5 \%$, and a roughness exponent of 1 . We also assume an RSR of $100 \mathrm{pm}$.

Another important question is roughness requirements into the future. Figure 6 shows a plot of the computed LWR for future nodes (increasing NA and decreasing feature size at a fixed $k_{1}$ factor). We assume a partial coherence of 0.5 , flare of $5 \%$, a roughness exponent of 1 , and an RSR of $100 \mathrm{pm}$. The defocus values are scaled as $1 / \mathrm{NA}^{2}$. We come to the surprising conclusion that the roughness problem becomes less severe into the future [10]. This is a result of having made the assumption that the RSR correlation length remains fixed into the future as the NA is increased causing us to moving further away from the peak seen in Fig. 5.

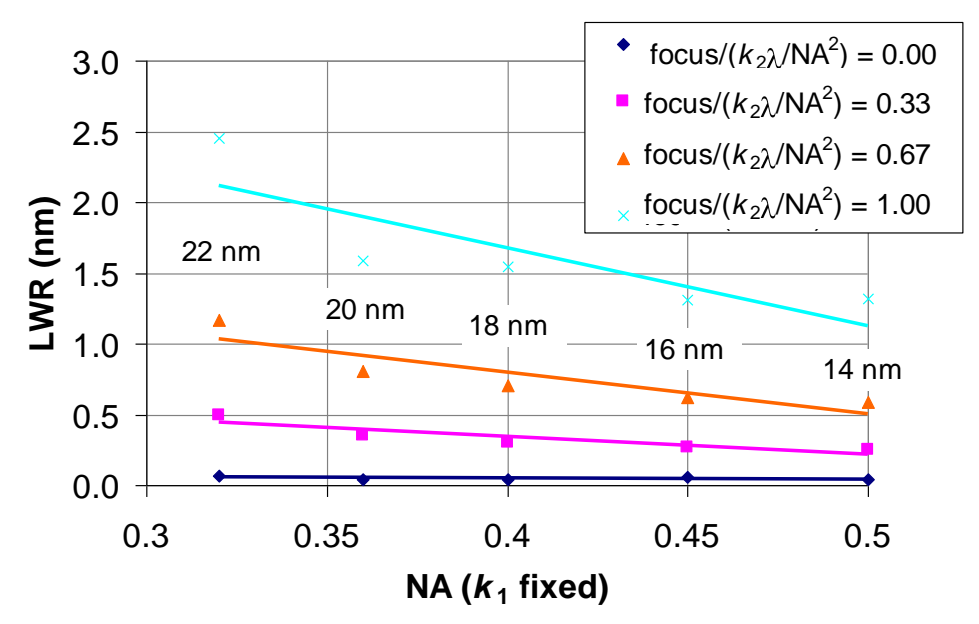

Fig. 6. Computed LWR for future nodes (increasing NA and decreasing feature size at a fixed $k_{1}$ factor). Partial coherence of 0.5 , flare of $5 \%$, roughness exponent of 1 , and RSR of $100 \mathrm{pm}$ of assumed. The defocus values are scaled as $1 / \mathrm{NA}^{2}$. 


\section{RSR METROLOGY}

Clearly RSR is an issue of concern which begs the question of how it can be measured. Currently, mask roughness is measured using atomic force microscopy (AFM), however, it is evident that AFM can only measure the top surface and not the true RSR. The true RSR, however, can be estimated by way of EUV scattering measurements [17]. Direct comparison of top surface AFM measurements to EUV scattering measurements (XRS) are shown in Fig. 7. The EUV scattering measurements were performed at the Center for X-ray Optics EUV Calibrations and Standards beamline at the Advanced Light Source [18]. In this case a variety of samples with increasing roughness were compared. At large roughness values, we find the AFM and XRS to match well, but as the roughness drops below approximately $0.4 \mathrm{~nm}$, the two methods start to differ significantly. Noting that all EUV masks of commercial relevance currently have roughness values of smaller than approximately $150 \mathrm{pm}$, we see that AFM roughness cannot be assumed to be an accurate metric.

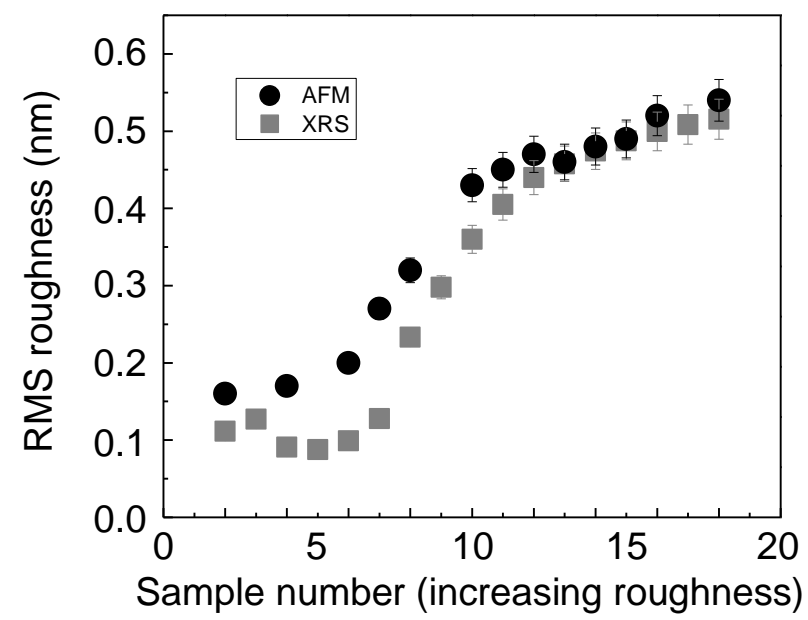

Fig. 7. Direct comparison of top surface AFM measurements to EUV scattering measurements (XRS). A variety of samples with increasing roughness are compared. At large roughness values, the AFM and XRS measurement match well, but as the roughness drops below approximately $0.4 \mathrm{~nm}$, the two methods start to differ significantly.

\section{CAPPING LAYER ROUGHNESS}

The analysis above implicitly assumed the phase roughness to be a result of RSR. Although RSR is the physical roughness source which mostly strongly couples to phase roughness, other sources of roughness can also be important, for example multilayer capping layer roughness. Unlike RSR, capping layer roughness couples to phase roughness through refraction and additionally causes reflected amplitude roughness, thus the optical properties of the capping layer must be taken into account. The most common capping layer for EUV mask multilayer stacks is ruthenium which provides a double-pass phase shift of $6^{\circ}$ per nm of thickness [19]. Additionally, $1 \mathrm{~nm}$ of ruthenium attenuates the light by $3 \%$ in double pass. By contrast, silicon would provide only $0.0036^{\circ}$ phase shift per nm of material double pass and $0.3 \%$ attenuation [19]. From this perspective, ruthenium does not appear to be an optimal choice for capping layer. Nevertheless, as-deposited ruthenium capping layers are more than adequately smooth for this not to be an issue.

The concern with the ruthenium capping layer arises when the mask is cleaned and the capping layer is potentially roughened. Figure 8 shows measured ruthenium capping layer roughness as a function of cleaning cycles using a conventional wet cleaning process [20]. Also shown is a linear fit to the data, extrapolating the damage out to 30 cleaning cycles. This roughness data can then be used to predict the resulting LWR, but as discussed above we must also account for the roughness correlation length and roughness exponent. For the cleaning process considered here, these values have been found to be $15 \mathrm{~nm}$ and 0.65 , respectively [10]. From these values, the resulting LWR can be computed (Fig.9), yielding near $1 \mathrm{~nm} \mathrm{LWR}$ at the edge of focus after 30 cleaning cycles. The asymmetric through focus behavior is a result of combined phase and amplitude roughness in the reflected field owing to the absorptive properties of the ruthenium. 

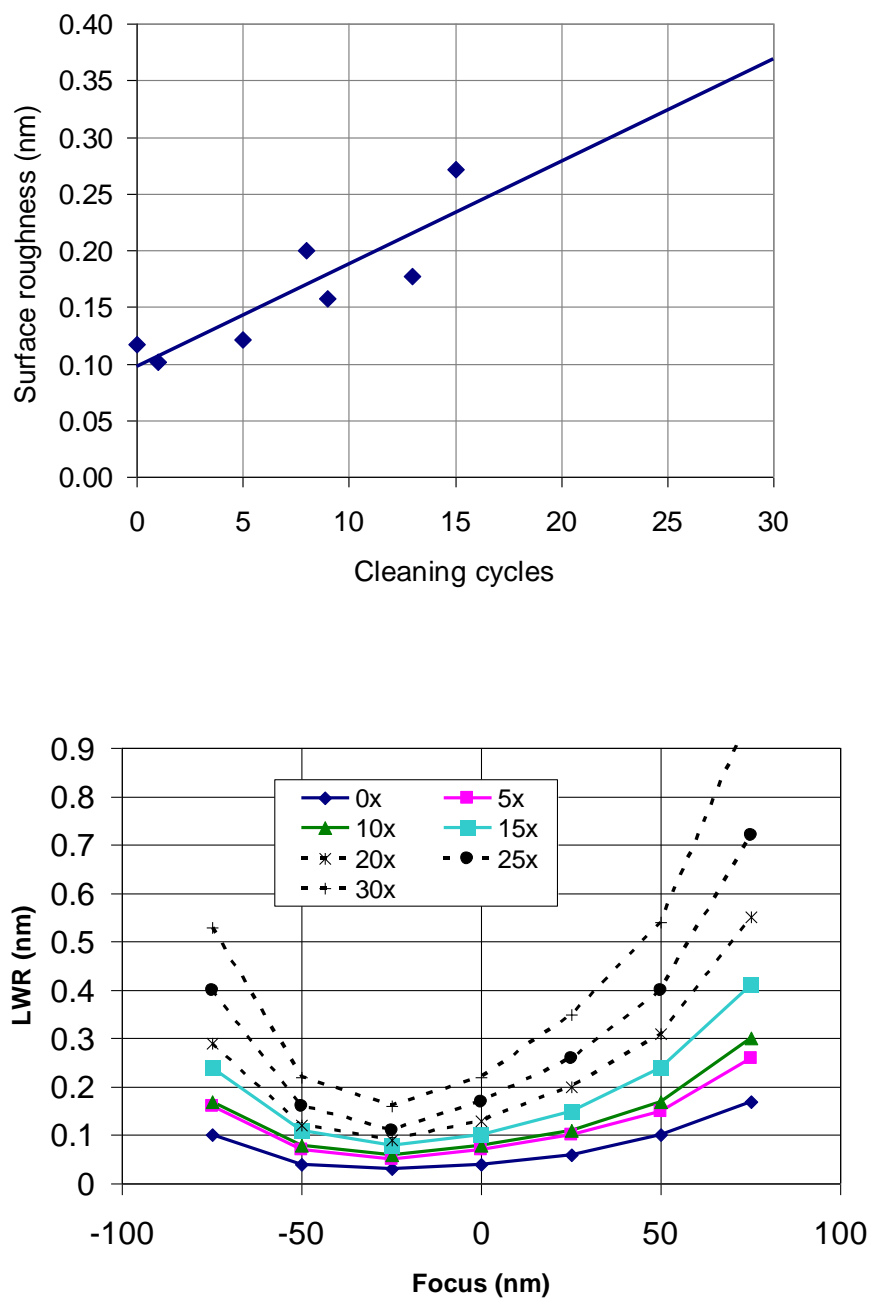

Fig. 8. Measured ruthenium capping layer roughness as a function of cleaning cycles using a conventional wet cleaning process [19]. Also shown is a linear fit to the data, extrapolating the damage out to 30 cleaning cycles.
Fig. 9. Computing LWR resulted from cleaning-induced capping layer roughness.

\section{SUMMARY}

Mask RSR is a potentially significant source of image plane LWR in EUV lithography. The sensitivity of the induced LWR to roughness depends on many factors including illumination partial coherence, defocus, roughness correlation length, roughness exponent, and NA. The strong dependence of LWR on the roughness correlation length is of particular interest and demonstrates the importance of specifying the higher order statistics of the mask roughness. Metrology of the mask roughness is also an issue of concern. Results show top surface AFM to not be a reliable metric, suggesting that actinic metrology is required to measure the RSR. Capping layer roughness has also been shown to be an issue of potential concern in light of cleaning induced damage of the ruthenium.

\section{ACKNOWLEDGEMENTS}

The authors are greatly indebted to CXRO MET team including Chris Anderson, Paul Denham, Gideon Jones, Lorie-Mae Baclea-An, and Nate Smith. This work was funded by SEMATECH and we thank Dominic Ashworth, Bryan Rice, and Stefan Wurm for continued support of the SEMATECH MET exposure facility at the Advanced Light Source. 
The work was performed at Lawrence Berkeley National Laboratory's Advanced Light Source synchrotron facility and was supported by SEMATECH through the U.S. Department of Energy under Contract No. DE-AC02-05CH11231.

\section{REFERENCES}

1. C. Wagner, et al., "EUV lithography at chipmakers has started: performance validation of ASML's NXE:3100," Proc. SPIE 7969, 79691F (2011).

2. F. Goodwin, T. Nakajima, P. Kearney, J. Kageyama, V. Jindal, M. Kishimoto, C. Lin, A. Ma, M. Godwin, and J. Harris-Jones, "SEMATECH's EUVL Mask Blank Defect Reduction Program: ML Deposition Defect Sources and Mitigation Strategies," 2010 International Symposium on Extreme Ultraviolet Lithography, Kobe, Japan, October 1820, 2010, proceedings available from SEMATECH, Austin, TX.

3. E. Gallagher, G. McIntyre, S. Raghunathan, L. Kindt, J. Whang, M. Barrett, T. Wallow, and O. Wood, "EUV masks under exposure: practical considerations," Proc. SPIE 7969, 79690W (2011).

4. N. Beaudry, T. Milster, "Effects of mask roughness and condenser scattering in EUVL systems," Proc. SPIE. 3676, 653-662 (1999).

5. P. Naulleau, "The relevance of mask-roughness-induced printed line-edge roughness in recent and future EUV lithography tests," Appl. Opt. 43, 4025-4032 (2004).

6. P. Naulleau, D. Niakoula, G. Zhang, "System-level line-edge roughness limits in extreme ultraviolet lithography," J. Vac. Sci. \& Technol. B 26, 1289-1293 (2008).

7. P. Naulleau and S. George, "Implications of image plane line-edge roughness requirements on extreme ultraviolet mask specifications," Proc. SPIE 7379, 73790O-73790O-11 (2009).

8. P. Naulleau, "Correlation method for the measure of mask-induced line-edge roughness in extreme ultraviolet lithography," Appl. Opt. 48, 3302-3307 (2009).

9. P. Naulleau, C. Anderson, P. Denham, S. George, K. Goldberg, B. Hoef, C. Koh, B. La Fontaine, W. Montgomery, J. Roller, T. Wallow, S. Wurm, "The SEMATECH Berkeley microfield exposure tool: learning at the 22-nm node and beyond," Proc. SPIE 7271, 7271W (2009).

10.P. Naulleau, B. McClinton, K. Goldberg, I. Mochi, and A. Rastegar, "Mask roughness challenges in extreme ultraviolet mask development," J. Vac. Sci. \& Technol. B, to be published (2011).

11. P. Naulleau and S. George, "Validity of the thin mask approximation in extreme ultraviolet mask roughness simulations," Appl. Opt., to be published (2011).

12. J. W. Goodman, Statistical Optics, John Wiley and Sons, New York, 1985, Chap. 7, 286-360

13. M. Teague, "Irradiance moments: their propagation and use for unique retrieval of phases," J. Opt. Soc. Am. $\mathbf{7 2}$, 1199-1209 (1982)

14. N. Striebl, "Phase imaging by the transport of intensity equation," Opt. Commun. 49, 6-10 (1984).

15. K. Ichikawa, A. Lohmann, and M. Takeda, "Phase retrieval based on the irradiance transport equation and the Fourier transport method," Appl. Opt. 27, 3433-3436 (1988).

16. K. A. Goldberg, I. Mochi, P. P. Naulleau, H.-S. Han, S. Huh, "Benchmarking EUV mask inspection beyond 0.25 NA,"SPIE Photomask (BACUS) 7122, 71222E-1 (2008)

17. S. George, P. Naulleau, I. Mochi, F. Salmassi, E. Gullikson, K. Goldberg, E. Anderson, "Replicated mask surface roughness effects on EUV lithographic patterning and line-edge roughness," Proc. SPIE 7969, 79690E (2011).

18. E. Gullikson, S. Mrowka, B. Kaufmann "Recent Developments in EUV Reflectometry at the Advanced Light Source," Proc. SPIE 363, (2001)

19. CXRO X-ray Materials Database (http://henke.lbl.gov/optical_constants/)

20. A. Rastegar, S. Eichenlaub, A. John, B. Lee, M. House, S. Huh, B. Cha, H. Yun, I. Mochi, and K. Goldberg, Proc. SPIE 7636, 76360N (2010). 


\section{DISCLAIMER}

This document was prepared as an account of work sponsored by the United States Government. While this document is believed to contain correct information, neither the United States Government nor any agency thereof, nor The Regents of the University of California, nor any of their employees, makes any warranty, express or implied, or assumes any legal responsibility for the accuracy, completeness, or usefulness of any information, apparatus, product, or process disclosed, or represents that its use would not infringe privately owned rights. Reference herein to any specific commercial product, process, or service by its trade name, trademark, manufacturer, or otherwise, does not necessarily constitute or imply its endorsement, recommendation, or favoring by the United States Government or any agency thereof, or The Regents of the University of California. The views and opinions of authors expressed herein do not necessarily state or reflect those of the United States Government or any agency thereof or The Regents of the University of California. 